\title{
Novel Position Estimation USING DIFFERENTIAL TIMING INFORMATION FOR ASYNCHRONOUS LTE/NR NETWORKS
}

\author{
Muhammad Abugabal, Yasmine Fahmy and Hazim Tawfik \\ Department of Electronics and Electrical Communications, \\ Cairo University, Cairo, Egypt
}

\begin{abstract}
Positioning techniques have been a common objective since the early development of wireless networks. However, current positioning methods in cellular networks, for instance, are still primarily focused on the use of the Global Navigation Satellite System (GNSS), which has several limitations, like high power drainage and failure in indoor scenarios. This study introduces a novel approach employing standard LTE signaling in order to provide high accuracy positioning estimation. The proposed technique is designed in analogy to the human sound localization system, eliminating the need of having information from three spatially diverse Base Stations (BSs). This is inspired by the perfect human $3 D$ sound localization with two ears. A field study is carried out in a dense urban city to verify the accuracy of the proposed technique, with more than 20 thousand measurement samples collected. The achieved positioning accuracy is meeting the latest Federal Communications Commission $(F C C)$ requirements in the planner dimension.
\end{abstract}

\section{KEYWORDS}

Cellular Networks, LTE, OTDoA, Sound Localization \& Wireless Positioning.

\section{INTRODUCTION}

The desire for wireless devices' positioning has existed since the introduction of commercial mobile networks. It has been further enforced by the US Federal Communications Commission (FCC) in 2001, mandating all wireless carriers to position users conducting emergency calls with a defined accuracy limits [1]. This requirement has evolved to reach a maximum planner error of 50 meters for $80 \%$ of the positioning attempts and maximum error of 5 meters in the vertical plane, to be achieved by mobile operators by 2021 [2]. The maximum vertical error requirement has evolved further to reach 3 meters for future applications [3]. These regulations have accelerated the development of various algorithms to estimate the location of wireless devices either in a given coordinate system or relative to network elements. Applications of cellular positioning are not limited to emergency requirements, but span other multiple use cases. For mobile carriers, anonymous users' positioning allows conduction of performance assessment and enhancement based on geographical grids rather than on cell basis. Positioning algorithms are classified into two main categories, the first is known as range-based positioning algorithm, where measurements associated with wireless environment aids the location estimate. Typical measurement methods include Global Positioning System (GPS), Time of Arrival (TOA), Angle of Arrival (AoA) and Received Signal Strength (RSS). The second category is known as rangefree positioning algorithms, utilizing network connectivity for localization [4].

Range-based positioning algorithms are not generally able to position devices accurately because of the induced measurement error associated with the radio environment. This error results from 
International Journal of Computer Networks \& Communications (IJCNC) Vol.13, No.4, July 2021

multi-path propagation, mobility and Non-Line of Sight (NLOS) propagation. Range-free positioning algorithms, on the contrary, provide better accuracy but require an initial phase to acquire trained data. The slow adoption of positioning algorithms stems from the low accuracy of range-based algorithms and the cost associated with range-free algorithms to acquire trained data. In this paper a novel approach is proposed to enhance the range-based positioning accuracy and applicability for a defined sample of devices, and can be further utilized as a basis for range-free methods to position the full population. The contributions of this paper are two-fold:

- The development of a generalized approach utilizing standard Long Term Evolution (LTE) measurements between transmitters and receivers without the need for extra functionalities, which enables wide adoption of the proposed positioning approach.

- Planner position estimation employing data from only two spatially separated Base Stations (BS), as opposed to typical planner positioning using at least three spatially separated BSs.

The remainder of the paper is organized as follows, section two provides a literature review focusing on identifying measurement quantities available in LTE systems followed by a description of the most common positioning algorithms for LTE networks. The proposed differential position estimation algorithm is described in section three, while in section four, results of the field trial are provided, followed by a conclusion in section five.

\section{Literature REVIEW}

\subsection{LTE Physical Layer Measurement}

Long-term Evolution (LTE) is a well-known broadband standard issued by 3GPP [5]. It meets the $4^{\text {th }}$ generation requirements and is currently contributing to the majority of broadband connections worldwide. LTE is utilizing primarily OFDM technology to provide high-speed connections to multiple mobile users simultaneously. It is available in both Frequency Division Duplex (FDD) and Time Division Duplex (TDD), with the earlier being dominant worldwide, while the latter is used in few countries in Asia like China. LTE standard procedures involve multiple measurements to describe the radio environment and aid to provide reliable communication. While physical layer measurements are designed to sustain communication between transmitter and receiver, like reporting received power, synchronization, channel estimation, among others, they can be utilized to estimate the user's position.

Reference Signal Received Power (RSRP) is a fundamental quantity in LTE measuring the received power of the reference signal in the downlink [6]. Timing Advance (TA) represents the amount of time the Mobile Station (MS) needs to advance its uplink transmission in order to reach the Base Station (BS) at the beginning of each slot, which ensures that transmissions from all users in the same cell arrive at the same time. The timing advance is maintained through a timing advance command sent regularly from the BS towards each MS. Timing advance commands are typically sent for connected users every few milliseconds. The LTE standard specifies a granularity for signalling timing advance of $0.52 \mu$ s which corresponds to a distance between BS and MS of 78.12 meters approximately [7]. TA and RSRP are basic parameters in LTE and are available in all LTE networks. Another common LTE measurement quantity is the Pre-Coder Matrix Index (PMI), which reflects the channel conditions and is signalled from the user to the cell. 
International Journal of Computer Networks \& Communications (IJCNC) Vol.13, No.4, July 2021

For New Radio (NR) system the same parameters exist either for non-standalone and standalone deployment scenarios. LTE quantities of the anchor cell are available for the earlier while the latter provides the NR parameters only [8].

\subsection{LTE Position Estimation Techniques}

Cellular positioning in LTE involves multiple methods developed over time. The most basic method is the Cell ID (CID) positioning, where a location server named Evolved Serving Mobile Location Centre (E-SMLC) requests the serving cell ID of the user from the Mobile Management Entity (MME), then it looks up a predefined database of the cell serving geographical area. The cell serving area is typically a polygon with size depending on many factors like cell type; outdoor or indoor, area nature; Urban or Suburban, and the frequency band used. Cell serving area increases with the use of lower frequency bands. In urban areas, it is typical to have an interbase station distance from 100 to 500 meters, while in suburban it can go up to a few kilometers.

The Enhanced CID (E-CID) positioning method improves the accuracy of CID through employing additional sources of information associated with the serving cell ID like TA, RSRP, and Angle of Arrival (AoA). It specifies a smaller geographical area than CID. The use of timing advance, for instance, specifies a circular contour for an Omni-directional cell and an arch for a directional cell. The use of signal strength adds additional information as well through matching the received power from serving cells and neighbours with the empirical propagation model. Angle of Arrival (AoA), when combined with timing advance, specifies a smaller part on the circular contour as a potential position of the user [9]. While the introduction of timing advance narrows down the polygon with the potential user location, it is contaminated with the multi-path environment especially in NLOS scenarios. Accordingly, the distance between MS and BS is reported higher than the true distance because of the longer propagation distance. This error is strongly influenced by the environmental aspects in the cell serving area. On the other hand, received signal strength is highly affected by multipath fading and users' mobility. The comparison with empirical propagation model will not provide descent accuracy in accordance because of the difficulty to acquire accurate digital maps and the environment dynamics. AoA in combination with TA should be able to improve the positioning accuracy. However, to acquire AoA, each cell should be equipped with multiple antenna arrays spatially separated. Most commercial mobile networks today don't use this setup and rather configure each cell with a cross polarized dual antenna arrays. This is due to change in the future when higher order antenna arrays and massive MIMO become available.

Another range-based algorithm standardized by 3GPP is the Time Difference of Arrival (TDoA), where either multiple BSs observe the difference in arrival time of the target MS, or the MS is observing the arrival time difference of multiple BS signals in the downlink. The latter case is more relevant in LTE networks because of the continuous broadcast channel transmitting from each cell. It is referred to as Observed Time Difference of Arrival (OTDoA) [10]. OTDoA is based on measurement of the differences in time of arrival of the Positioning Reference Signal (PRS) of multiple cells spatially separated. A comparison between two cells specifies a hyperbola identifying a potential user location. For planner positioning at least measurements from three BSs are needed. UTDoA is almost similar to OTDOA with the exception that the BSs, on the contrary, observe the differences in time of arrival of uplink the signal transmitted by the user [11]. Accuracy of OTDoA algorithm has been verified using real measurements in LTE network [12], spanning a range from 20 to 63 meters based on the environment and can achieve an average accuracy of $50 \mathrm{~m} \mathrm{[13].} \mathrm{It} \mathrm{is} \mathrm{considered} \mathrm{one} \mathrm{of} \mathrm{the} \mathrm{high} \mathrm{accuracy} \mathrm{positioning} \mathrm{algorithms} \mathrm{in}$ mobile networks which don't require synchronization between transmitter and receiver, and support indoor localization as well [14]. On the other hand, it requires tight time and phase synchronization of all Base Stations in the target region, which is the primary reason why it is not 
commonly deployed in current FDD mobile networks. A time difference of $100 \mathrm{~ns}$ between different $\mathrm{BSs}$ is equivalent to error in positioning of around $30 \mathrm{~m}$ [15]. The increasing deployment of 5G in TDD spectrum will facilitate the adoption of OTDoA because of the tight time and phase synchronization deployed to allow the TDD technology. Another limitation comes from the necessity to implement additional protocol and server in mobile networks to support the location protocol which adds to the complexity and cost.

It is well established that one of the most accurate positioning algorithms is the GPS. It is considered a range-based technique where a satellite signal is utilized to precisely position the wireless receiver. For cellular networks an assisted GPS algorithm was introduced by a company named SnapTrack [16] in order to aid mobile devices in GPS positioning. It was also introduced in 3GPP standards for all technologies [17], where the network aids the mobile device by accelerating signal acquisition and reducing battery requirements accordingly. Furthermore, to facilitate mobile network management and quality of service assurance by mobile carriers, 3GPP has introduced a functionality called Minimization of Drive Tests (MDT) where it allows the Mobile Station (MS) to share its GPS coordinates with the operating carrier network [18]. While GPS based positioning provides almost the best accuracy [13], it is not widely used by mobile carriers for the purpose of network management and quality of service assurance due to many reasons; first the MS must be in LOS with the satellites, limiting the position reporting to the outdoor scenarios, second ordering mobile devices to share their GPS position imposes privacy constraints, third faster battery drainage with frequent requests of GPS positions is noticed [19].

A comparison of the performance of different position estimation algorithms can be found in [13]. Yet with the limited adoption of positioning algorithms by mobile operators, the motivation to develop a positioning algorithm that can be globally applicable to most common LTE/NR networks arises [13]. Up to the author's knowledge most recent positioning techniques' development is focused around two categories, the first involves the use of finger printing inherited from its proven general accuracy [21] and [22], while the other category utilizes the increased usage of massive MIMO in 5G networks [23] and its capability of inclusion of beam angle in the positioning algorithm. Massive MIMO even allows the distinction between LOS and NLOS components. However, the cost associated with the training phase in fingerprinting, and the non-existence of network-wide deployment of massive MIMO brings the need to enhance the range-based positioning techniques accordingly.

\section{Differential Estimation of Position}

Consider a Mobile Station (MS) connected to an LTE cell $1_{1}$ belonging to a base station $\mathrm{BS}_{1}$. Since cell $l_{1}$ is the serving cell, timing advance $\tau_{1, i}$ is continuously updated throughout the connection in order to maintain the slot synchronization. The quantity $T A_{1, i}$ reflects the measured range $\tilde{d}_{1, i}$ between the MS and cell $1_{1}$, whereas the true range is denoted by $d_{1, i}$, and $i$ represents the measured sample and belongs to $[0,1,2, \ldots \mathrm{N}]$, where $\mathrm{N}$ is the total number of observations. The relation between $\tilde{d}_{1, i}$ and $d_{1, i}$ depends on the connection scenario being LOS or NLOS as follows

$$
\begin{gathered}
\tilde{d}_{1, i}=d_{1, i}+z_{\text {los }_{1, i}}+q_{1, i} \text { LOS scenario, } \\
\tilde{d}_{1, i}=d_{1, i}+b_{1, i}+z_{\text {nlos } 1, i}+q_{1, i} \text { NLOS scenario, }
\end{gathered}
$$

where $z_{\text {los }_{1, i}}$ and $z_{n l o s_{1, i}}$ represent the measurement noise sample and are modeled as zero mean normal random variable with variances of $\sigma_{z_{\text {los }}}$ and $\sigma_{z_{n l o s}}$ respectively, $q_{1, i}$ is the error term 
introduced due to the quantization of the timing advance, and is thus modelled as uniformly distributed random variable over the period $[-39.06,39.06]$ meters. $b_{1}$ is a positive bias arising from NLOS propagation and is modeled as exponential distribution with mean $1 / \lambda_{b_{1}}$ and variance $1 / \lambda_{b_{1}}{ }^{2}$. The probability distribution functions of all the error sources are given by the following equations for both LOS and NLOS scenarios:

$$
\begin{aligned}
& f\left(z_{l o_{1}}\right)=\frac{1}{2 \pi \sigma_{z_{l o s_{1}}}^{2}} \exp \left[-\frac{z_{l o s_{1}}^{2}}{2 \sigma_{z_{l o s_{1}}}^{2}}\right], \text { LOS } \\
& f\left(z_{\text {nlos }_{1}}\right)=\frac{1}{2 \pi \sigma_{z_{n l o s_{1}}}^{2}} \exp \left[-\frac{z_{\text {nlos }}^{2}}{2 \sigma_{z_{\text {nlos }}}^{2}}\right], \text { NLOS, } \\
& f\left(q_{1}\right)=\left\{\begin{array}{c}
\frac{1}{78.125},-39.062 \leq q_{1} \leq 39.062, \\
0, \text { otherwise }
\end{array}\right. \\
& f\left(b_{1}\right)=\left\{\begin{array}{c}
\lambda_{b_{1}} e^{-\lambda_{b_{1} b_{1}}}, b_{1} \geq 0 . \\
0, b_{1}<0
\end{array}\right.
\end{aligned}
$$

The information of $\tau_{1, i}$ only provides a circular contour with the potential user location. Information from at least three other cells is needed to further locate the user in the planner dimension. In the proposed algorithm information from two spatially separated cells only is employed for planner localization. A problem arises from having the timing advance available from the serving cell only. To acquire timing advance for the neighbouring cells, it is proposed to limit the positioning estimates around the events of handovers provided that MS is stationary or quasi-stationary. This approach allows the acquisition of the timing advance from the source cell before the handover and the timing advance of the neighboring cell after the handover. It is worth noting that MS stationarity and handover are not mutually exclusive events. Frequent handovers happen while mobile users are stationary because of multi-path propagation and dynamic channel conditions. The proposed stationarity condition implies that the user is alternating between the maximum of three timing advance values. This has been set after conducting field measurements, as detailed in a results section, to achieve a compromise between user mobility state accuracy and allowing measurement noise while the user is stationary.

Timing advance $\tau_{1, i}$ and $\tau_{2, i}$ are observed before and after handover between cell ${ }_{1}$ and cell $_{2}$ respectively. The knowledge of timing advance values allows only an ambiguous positioning attempt using trilateration for instance as described in [4]. Typically, information from three cells is needed to reduce ambiguity. Trilateration positioning is based on intersecting the circular ranges defined by each timing advance quantity. Two problems are arising accordingly; first, error probability specified by equations (3) to (6) is relatively high compared to the 50 meters' requirements of FCC especially for NLOS scenarios, where the bias is having a non-zero mean. Second, the ambiguity in position estimation resulting from observing information from two cells only, where it is very unlikely to acquire information from a third cell through a handover event without spatial movement of the user. An algorithm is developed to provide high accuracy position estimate using information from two cells only, inspired by the perfect 3D positioning capabilities of human dual ears [20]. In human acoustic positioning system, the first attempt in positioning employs a comparison of sound reaching the two ears in terms of amplitude and phase. Accordingly, attention will be made to the differential timing advance rather than examining each value of timing advance through trilateration for instance. The differential measured range, $\Delta \tilde{d}_{i}=\tilde{d}_{1, i}-\tilde{d}_{2, i}$, is given by 


$$
\begin{aligned}
& \Delta \tilde{d}_{i}=\Delta d_{i}+\Delta z_{\text {los }, i}+\Delta q_{i} \text { LOS } \\
& \Delta \tilde{d}_{i}=\Delta d_{i}+\Delta b_{i}+\Delta z_{\text {nlos }, i}+\Delta q_{i} \text { NLOS. }
\end{aligned}
$$

In urban cities, it is unlikely to have LOS scenarios. Moreover, it is difficult to have handover between cells in different LOS/NLOS scenarios. It is then assumed in the following analysis that the two cells involved in the handover are in NLOS scenario. Any mixed handover scenarios will be considered as an error in position estimation. Since $b_{1}$ and $b_{2}$ are independent and identically distributed exponential random variables, their differential probability density function is known to be a Laplace distribution as follows:

$$
f_{\Delta B}(\Delta b)=\frac{\lambda}{2} e^{|\lambda| \Delta b} .
$$

Where $\lambda$ is equal to $\lambda_{b 1}$ and $\lambda_{b 2}$ because of the identical distribution assumption. The main advantage achieved is a zero mean distribution on the expense of doubling the variance from $1 / \lambda^{2}$ to $2 / \lambda^{2}$, which is a common drawback in many cases such as using the trilateration for instance [4]. Similarly, the differential quantization error keeps the zero mean advantage while doubling the variance.

$$
\begin{aligned}
& f_{\Delta Q}(\Delta q)=\left\{\begin{array}{c}
\frac{1}{78.125}(1-|\Delta q|),-78.125 \leq \Delta q \leq 78.125 \\
0, \text { otherwise }
\end{array}\right. \\
& f_{\Delta Z_{l o s}}\left(\Delta z_{l o s}\right)=\frac{1}{4 \pi \sigma_{z_{l o s}}^{2}} \exp \left[-\frac{z_{l o s}^{2}}{4 \sigma_{z_{l o s}}^{2}}\right] \text {, }
\end{aligned}
$$

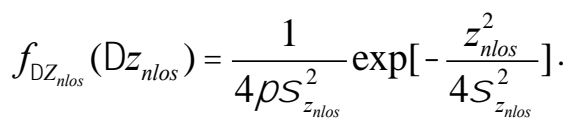

The differential range equations (7) and (8) have led to achieving zero mean error probabilities given by equations (9) through (12). This leads to the use of a minimum variance unbiased estimator. The differential TA formulation also defines a hyperbola with the potential user location. It is very unlikely in NLOS scenarios to have the actual range exceeding the measured range because of the positive bias induced by the reflections from multiple objects. Thus the information about the absolute measured range is proposed to set a constraint on the range. The Hyperbola by equations (7) and (8) extends to infinity, but with the constraints in (13) and (14) the potential user location on the Hyperbola will be limited to a finite range.

$$
\begin{aligned}
& d_{1}<\tilde{d}_{1}, \\
& d_{2}<\tilde{d}_{2} .
\end{aligned}
$$

Another important consideration to improve the accuracy of position estimation is to ensure that the user is in the intended cell serving area. Users served by overshooting cells exhibit more uncertainty in their position estimate, and hence the following condition should apply:

$$
\max \left(\tilde{d}_{1}, \tilde{d}_{2}\right)<d_{12},
$$


where $d_{12}$ is the distance between the base stations in the two cells. Figure 1 (a) illustrates the area enclosing the potential user location, where the MS is likely to be located in the hashed area. The trilateration method in Figure 1 (b) is not considering that the error is biased due to NLOS scenario. It assumes a zero mean error component in each measured range quantity.

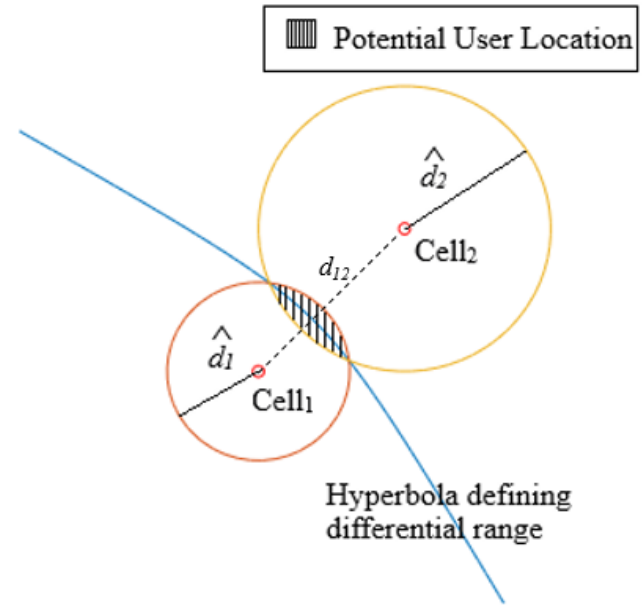

(a)

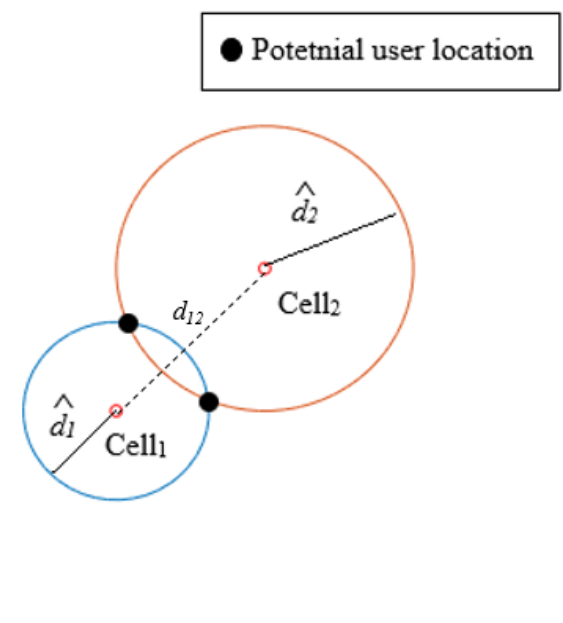

(b)

Figure 1. Position estimation techniques, proposed differential technique (a), Trilateration (b)

The handover event in LTE is controlled by the BS after evaluating the measured RSRP from both serving and target cells. The Handover takes place at a configurable signal strength difference between the serving and target cells, with a typical threshold in the range of $3 \mathrm{~dB}$. Thus the power levels of the two neighboring cells are comparable, which allows the setting of an additional condition to ensure that both cells exhibit the same conditions in either LOS or NLOS scenarios. Given that power levels are comparable, to ensure similar conditions, the differential range should be less than a threshold $\gamma$, which is designed to be 156 meters as detailed in section 4. If on the other hand the measured differential range is high, it implies that once a cell is having a more clear path to the user than the other, the condition for similar radio conditions is given by

$$
\left|\tilde{d}_{1}-\tilde{d}_{2}\right|<\gamma
$$

The uncertainty of user location within the probable area in Figure 1 (a) can be viewed as the cone of confusion problem within the Human acoustic location system. One of the techniques used by humans to resolve cone of confusion is head movement to collect more samples with different phase information [20], while this is not possible in cellular systems, an analogous approach would be to utilize information about signal strength received from neighboring cells located in the same BSs exhibiting the handover but oriented in a different azimuth through directional antennas. In contrary to trilateration, the intersection points between two measured range circles are not representing the probable user location because they exhibit zero bias in range measurement which is unlikely in NLOS scenarios. Inside the probable area of user location, it is more likely that the user is located in a point on the hyperbola inside the probable polygon based on zero mean error equations (9) through (12). RSRP measurements are available for serving cells as well as neighboring cells before and after handover. While RSRP measurements are available, the phase information is not. Moreover, the BSs might not be synchronized, which accordingly makes it difficult to establish probability estimation for the AoA based on measured RSRP of different cells. However, hypothesis testing is employed to 
select the most probable point for user location from a set of defined points. If MS is only reporting the serving cell RSRP, then the serving angle is assumed to be the angle of the highest antenna gain of the serving cell, whereas if measurements exist for neighboring cells at the same $\mathrm{BS}$, the strongest neighbor power $R S_{n, l}$ is examined about the power of the serving cells $R S_{l}$. If it is within $3 \mathrm{~dB}$, then the serving angle is assumed to be drifted toward the main beam of the neighboring cell, but with half weight, i.e. the serving angle $\theta_{l}$ is equal to the angle of the strongest beam of serving cells drifted towards the neighboring cell main beam angle $\varphi_{n, l}$ with an angle equals to quarter the difference between the two cells main beam angles. For differences higher than $3 \mathrm{~dB}$, the user is assumed to be served through the angle of the main beam of the serving cell $\varphi_{1}$. The same methodologies are applied after the handover. The estimated serving angle is used to select the user location from the three highlighted positions in Figure 2. The three points of the probable user location are designed to be first the mid-point, defined on the line joining the location of the two cells, then the other two points are located on the mid- distance between the mid-point and the intersection of the two measured range circles. A point is selected from the three- points to minimize the square error of the estimated serving angle before and after handover.

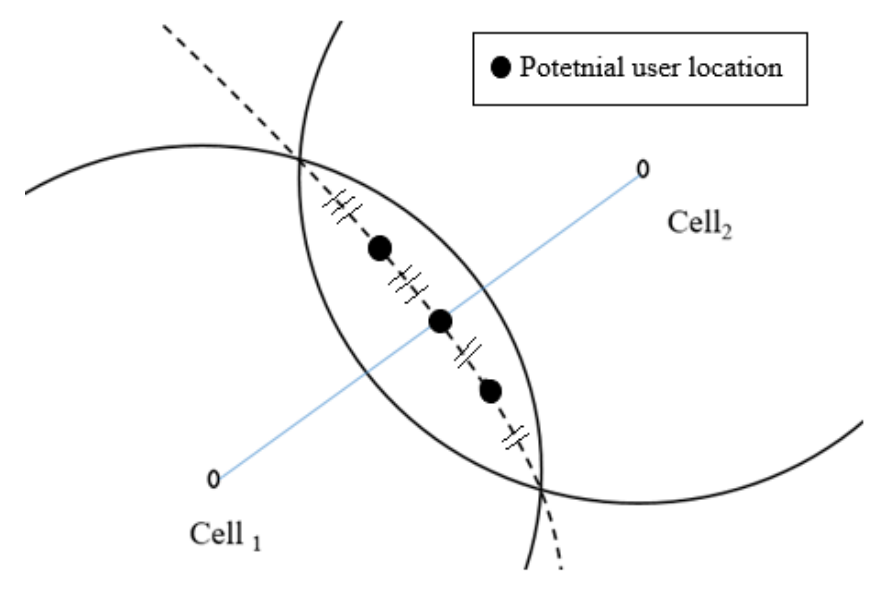

Figure 2. Potential user locations on the differential range hyperbola

Another way to ensure that the user is static or quasi-static is through examining the changes in TA during the observation period. Since each timing advance step is reflecting a range of $78.12 \mathrm{~m}$, then if the user exhibits more than two- step changes in timing advance it is regarded as a user with high mobility. The observation period is proposed to be dynamic as long as the user is oscillating between a maximum of three TA values. This allows more averaging of the measured range in order to reduce the noise. $\tau_{1, n}$ is the timing advance value of cell $1_{1}$ at instance $n, i$ is the number of measurements where user is considered static. In Algorithm 1, the proposed positioning algorithm is described. 


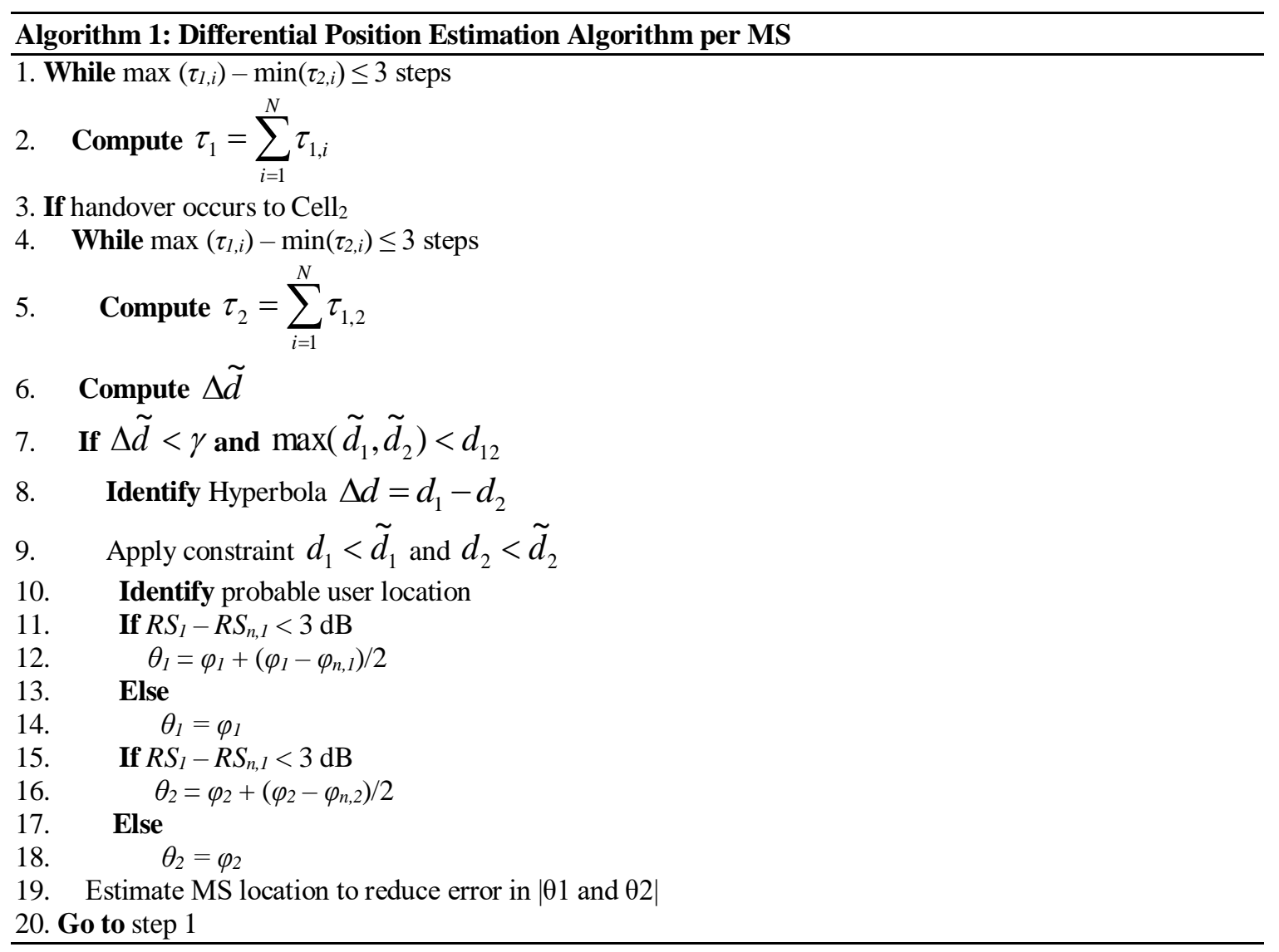

The algorithm is proposed to run in the Mobile Operators' Operations Support Systems (OSS) where all measurement reports from all users are collected in real time. It is best applied in urban environments where dense base stations are likely to have handovers even for quasi-stationary users.

\section{Measurements AND Results}

To test the newly proposed technique, real field measurements were collected in a highly density urban city using smartphones configured to log messages communicated with the network in both Uplink and Downlink. This allows access to physical layer measurements like TA and RSS among others. The frequency band configured is the $2100 \mathrm{MHz}$ band with $10 \mathrm{MHz}$ spectrum allocation on LTE technology. TA measurements are collected while conducting a drive route with a speed of less than $10 \mathrm{Kmph}$. The used phones have also the capability to log the GPS reading with an accuracy of less than three meters to serve as basis of comparison against the true location. The test area includes around 100 BSs. More than 20,000 measurement messages are collected. The average cell radius size is 350 meters. From the total number of handover events, $35 \%$ didn't meet the proposed positioning algorithm requirements, mainly due to overshooting cells. Few challenges have faced the experiment, resulting from the need to keep the car speed constant during the driving test and the requirement to provide accurate cell location for the algorithm. Other limitations include the necessity to conduct the drive route in an outdoor scenario to be able to acquire the GOS signal for comparison as well as the lack of accurate information about the RF coaxial cable length installed in towers which affect the propagated distance. 
The reporting of TA value is done every few milliseconds as shown for a sample in Figure 3, where it is observed that the measured range follows the true range with a positive bias resulting from NLOS propagation and oscillations resulting from the measurement noise. While the drive test is performed in a mobility state, a window of interest spans three seconds before and after the handover, which corresponds to displacement less than 5 meters for the given car speed.

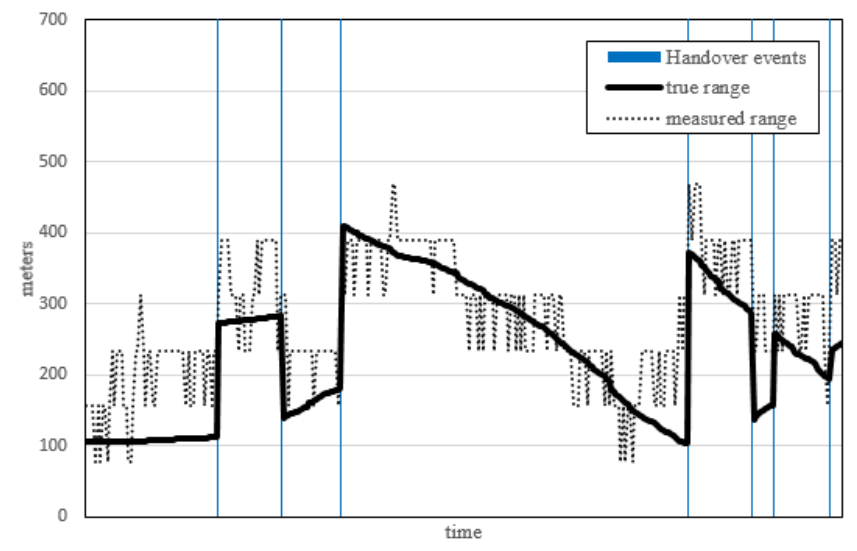

Figure 3. Sample of measured range against the corresponding true range

In Figure 4, the probability distribution function of the error between the measured distance $\tilde{d}$ and the real distance $d$ of the MS from the serving cell is plotted. It is clear from the figure that the error mean value is a non-zero value which is expected due to the multipath propagation effect in NLOS scenarios. The average value of the error delay in the test area is around 70 meters which reflects the environment propagation nature and it also embeds the granularity nature of the reported timing advance.

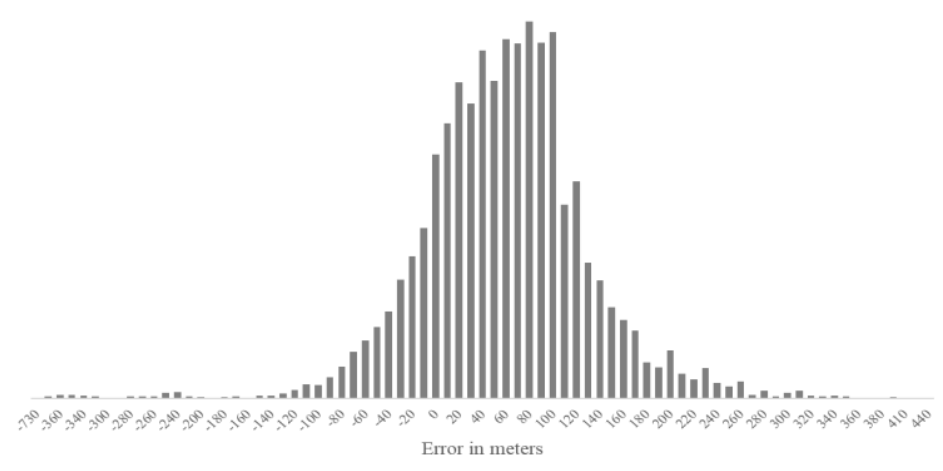

Figure 4. Error distribution of the measured range

Figure 5, an important note is that negative error only happens at small distances from the sites (less than 78 meters). When the distance increases the error positively increases as the effect of the multipath propagation increases. While the negative error appears, its magnitude is small and it is arising from the granularity measurement of their timing advance. Another contribution to the error is the deviation of the antenna geographical location against the archived cell location. One of the limitations in range-based position techniques is arising from the need of availing accurate cell and antenna physical information. When the MS gets farther from the cell, NLOS error becomes more dominant. The parameter $\gamma$ is designed to be 156 meters to ensure that error is contained within FCC requirements. 
International Journal of Computer Networks \& Communications (IJCNC) Vol.13, No.4, July 2021

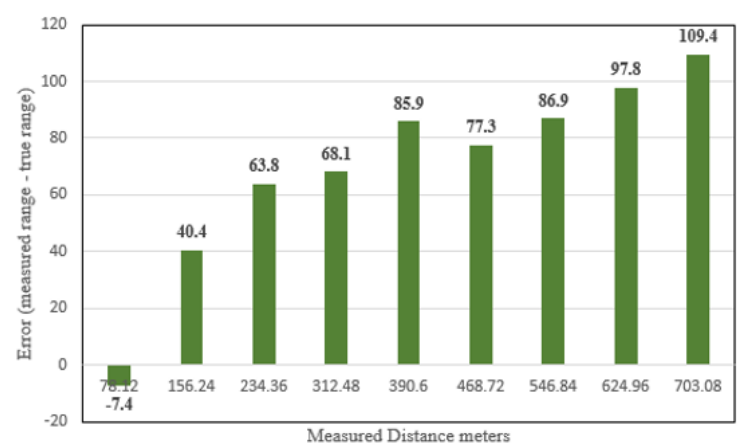

Figure 5. Error in range measurements against the measured range

The error in the measured differential range $(\Delta \tilde{d}-\Delta d)$ probability density function is plotted in Figure 6 . The zero mean together with the extended variance resulting from different sources of error is clear, following equations (9) through (12).

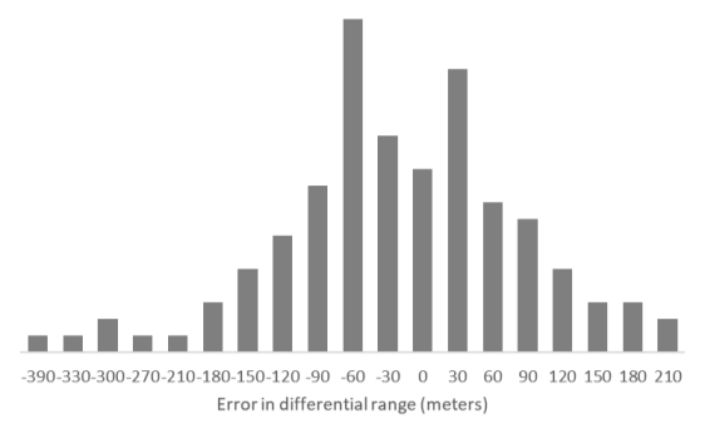

Figure 6. Error distribution of the measured differential range

In Figure 7, it is clear that the proposed algorithm is better matching the true range. The bias has been reduced and is showing the ability to be reduced further in future research.

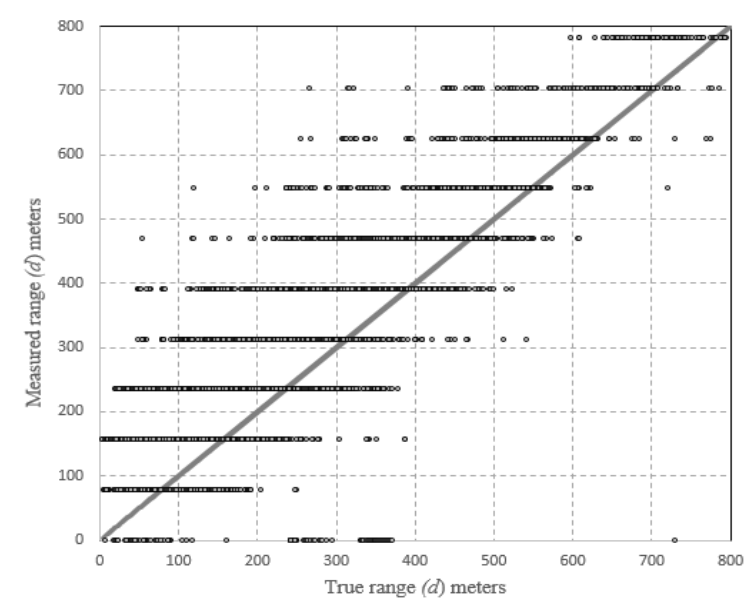

Figure 7. Scatter analysis of the measured range against the range 
The prime objective of the proposed positioning algorithm is to determine the most probable coordinates for user location. However, Figure 8 is representing the range of the estimated position relative to the true range. When compared to Figure 7, it is clear that the proposed algorithm is better matching the true range. The bias has been reduced and is showing the ability to be reduced further in future research.

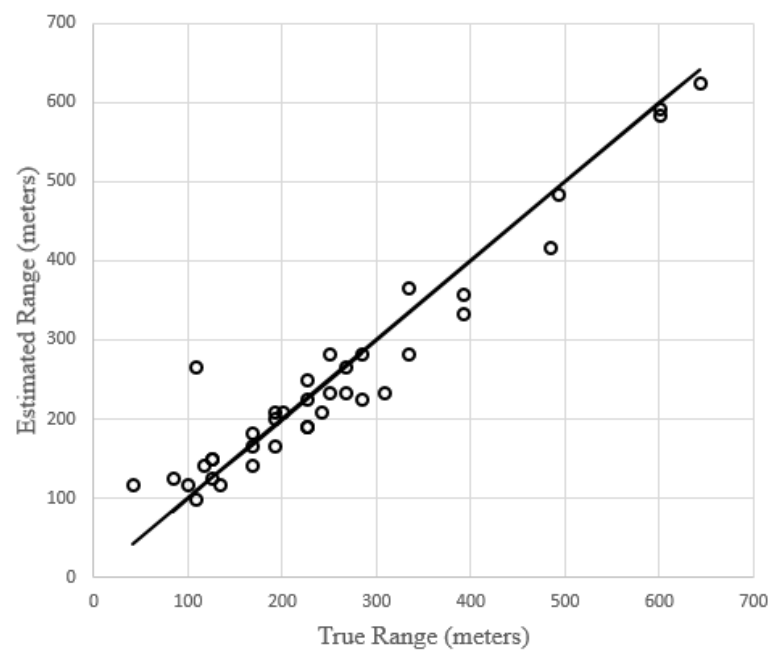

Figure 8. Scatter analysis of the estimated range against the true range

The estimated range error of the suggested algorithm $(\tilde{d}-d)$ is plotted in Figure 9 together with the measured range error $(\tilde{d}-d)$. The estimated distance is closer to the true distance than the measured distance, which results from the proposed noise reduction approach. While the estimated range is closer to the true range, it is generally slightly lower than the true range because of the assumption of high bias and avoidance of the measured range intersecting points. This is showing the possibility to even reduce the error further by expecting the bias order per area nature, which may in some occasions favor the measured range intersecting points, this requires field measurement from different area nature and stand on key differences

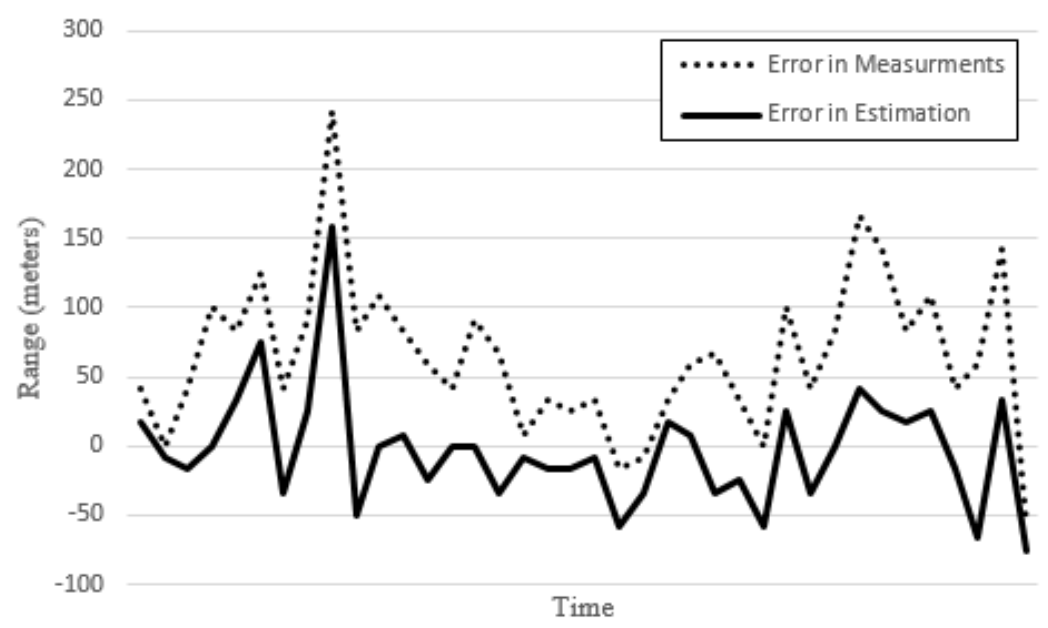

Figure 9. Sample tracking of estimated range error and measured range error 
In Figure 10, the distribution of absolute error values is plotted, with a mean measurement error of 70 meters, whereas the mean estimation error is 30 meters, showing the ability of the proposed algorithm to reduce the error for asynchronous LTE networks.

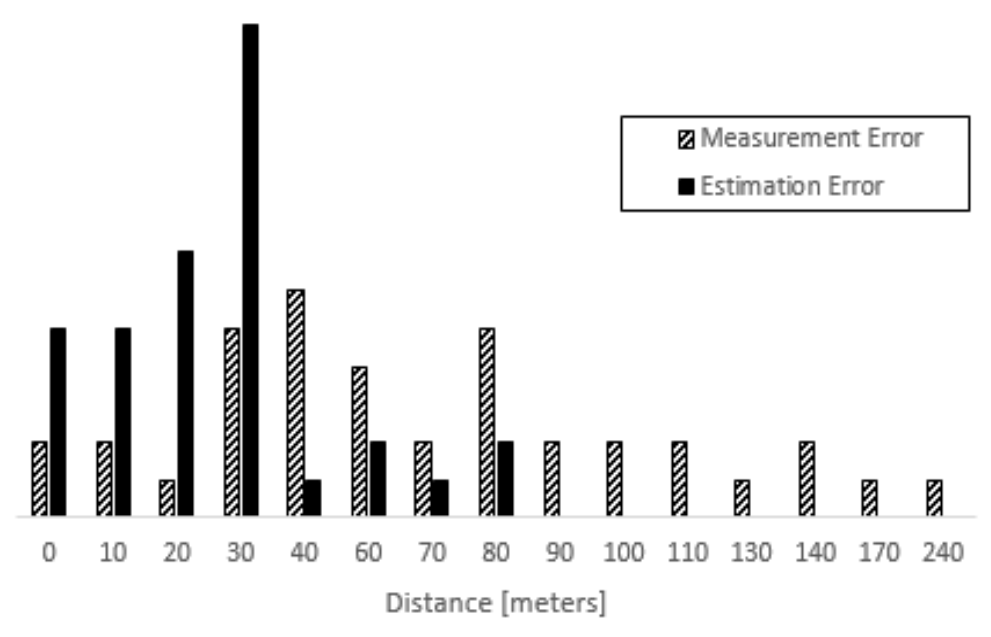

Figure 10. Absolute error distribution of the measured and estimated ranges

To conclude, applying the proposed differential estimation in the planner domain, a median error in position estimation is 40 meters, which is meeting the FCC requirements of position estimation of 50 meters for calls experiencing handover. The proposed technique is adequate in urban environments and specifies few assumptions for accurate positioning including the user quasistationarity and not being served from an overshooting cell.

The technique can be used to estimate the position of a target sample then can further be utilized to position the whole population using fingerprinting techniques. The proposed algorithm can be enhanced further by examining multiple frequency bands to improve the accuracy especially for the phones that support simultaneous carrier aggregation of different frequency bands.

\section{CONClusion}

The proposed positioning algorithm managed to achieve positioning accuracy less than 50 meters while using information from only two cells. It has the advantage of applying to all commercial LTE mobile networks without adding extra hardware. The main requirement to achieve this accuracy is to base the analysis on the handover events, followed by ensuring user stationarity and non-overshooting cells. While the accuracy might not be of high precision to serve the emergency applications, it is adequate for providing mobile operators with a useful insight into the quality of service per geographical grid. The typical performance management in commercial mobile networks today is primarily based on examining average cell performance as a whole. This algorithm is can leverage the performance management capabilities in mobile operators to provide better services on a grid basis.

\section{CONFLICTS OF INTEREST}

The author declares no conflict of interest. 
International Journal of Computer Networks \& Communications (IJCNC) Vol.13, No.4, July 2021

\section{REFERENCES}

[1] Djuknic, G. M., \& Richton, R. E. Geolocation and assisted GPS. Computer, 34 (3), 123-125, 2001.

[2] "PS Docket No. 07-114 wireless E911 location accuracy requirements," January 2015.

[3] FCC, "Wireless E911 Location Accuracy Requirements," Fifth Report and Order PS Docket 07-114., Apr. 2019.

[4] S. A. Zekavat and R. M. Buehrer, Handbook of Position Location. IEEE Press and Wiley, 2019.

[5] Astély, D., Dahlman, E., Furuskär, A., Jading, Y., Lindström, M., \& Parkvall, S. (2009). LTE: the evolution of mobile broadband. IEEE Communications magazine, 47 (4), 44-51.

[6] 3GPP TS 36.214, EUTRA; Physical layer; Measurements protocol specification (Rel.15), v.15.5.0. (2020-1).

[7] 3GPP TS 36.211 version 8.9.0 Release 8, "LTE; Evolved Universal Terrestrial Radio Access (EUTRA); Physical channels and modulation”, Apr. 2010.

[8] 3GPP TS 38.133 V15.8.0. "NR; Requirements for support of radio resource management”. Jan. 2020.

[9] Kangas, Ari, and Torbjorn Wigren. "Angle of arrival localization in \{LTE\} using \{MIMO $\}$ pre-coder index feedback." IEEE Communications Letters 17.8 (2013): 1584-1587.

[10] UE Positioning in E-UTRAN_-Stage 2, Release 13, document TS 36.305, 3GPP, Jan. 2016.

[11] Chen, Chih-Yu, and Wen-Rong Wu. "Three-dimensional positioning for LTE systems." IEEE Transactions on vehicular technology 66.4 (2016): 3220-3234.

[12] J. Medbo, I. Siomina, A. Kangas, and J. Furuskoh, "Propagation channel impact on LTE positioning accuracy: A study based on real measurements of observed time difference of arrival," in Proc. IEEE 20th Int. Symp. PIMRC, Tokyo, Japan, Sep. 2009, pp. 2213-2217.

[13] A. del Peral-Rosado, R. Raulefs, J. A. LÂs pez-Salcedo, and G. Seco- Granados, "Survey of cellular mobile radio localization methods: From $1 \mathrm{G}$ to 5G," IEEE Commun. Surveys Tuts., vol. 20, no. 2, pp. 1124- 1148, Second quarter 2018.

[14] Driusso, Marco, et al. "Indoor positioning using LTE signals." 2016 International Conference on Indoor Positioning and Indoor Navigation (IPIN). IEEE, 2016.

[15] J. A. del Peral-Rosado, J. A. López-Salcedo, F. Zanier, and G. Seco- Granados, "Position accuracy of joint time-delay and channel estima- tors in lte networks," IEEE Access, vol. 6, pp. $25185-25$ 199, 2018.

[16] M. Moeglein and N. Krasner, “An introduction to SnapTrack ${ }^{\circledR}$ server-aided GPS technology,” in Proc. ION GPS, pp. 333-342, Sept. 1998.

[17] 3GPP TS 25.305, "Stage 2 functional specification of user equipment (UE) positioning in UTRAN," Rel. 99, V3.0.0, Dec. 1999.

[18] Technical Specification Group Radio Access Network; Universal Terres- trial Radio Access (UTRA) and Evolved Universal Terrestrial Radio Access (E-UTRA); Radio measurement collection for Minimization of Drive Tests (MDT)_Overall Description, document 3GPP TS 37.320 v.14, 2011.

[19] Lo'ai, A. Tawalbeh, et al. "Greener and smarter phones for future cities: Characterizing the impact of GPS signal strength on power consumption." IEEE Access 4 (2016): 858-868.

[20] Risoud, M., et al. "Sound source localization." European annals of otorhinolaryngology, head and neck diseases 135.4 (2018): 259-264.

[21] Fang, W., Xie, C., \& Ran, B. (2020). An Accurate and Real-Time Commercial Indoor Localization System in LTE Networks. IEEE Access, 9, 21167-21179.

[22] Li, You, et al. "Toward robust crowdsourcing-based localization: A fingerprinting accuracy indicator enhanced wireless/magnetic/inertial integration approach." IEEE Internet of Things Journal 6.2 (2018): 3585-3600

[23] Mendrzik, R., Wymeersch, H., Bauch, G., \& Abu-Shaban, Z. (2018). Harnessing NLOS components for position and orientation estimation in $5 \mathrm{G}$ millimeter wave MIMO. IEEE Transactions on Wireless Communications, 18(1), 93-107. 